Pacific Journal of Mathematic 


\title{
ISOMETRIES OF CERTAIN FUNCTION SPACES
}

\author{
K. W. TAM
}

Let $X$ be a discrete symmetric Banach function space with absolutely continuous norm. We prove by the method of generalized hermitian operator that an operator $U$ on $X$ is an onto isometry if and only if it is of the form:

$$
U f(.)=u(.) f(T .) \text { all } f \in X,
$$

where $u$ is a unimodular function and $T$ is a set isomorphism of the underlying measure space. That other types of isometries occur if the symmetry condition is not present is illustrated by an example. We completely describe the isometries of a reflexive Orlicz space $L_{M \phi}\left(\neq L_{2}\right)$ provided the atoms have equal mass (the atom-free case has been treated by G. Lumer); similarly for the case that no Hilbert subspace occurs.

We shall reproduce some definitions and results from [4] which will be needed in the sequel.

Definition. Let $X$ be a vector space. A semi-inner-product on $X$ is a mapping [,] of $X \times X$ into the field of numbers (real or complex) such that

$$
\begin{aligned}
{[x+y, z] } & =[x, z]+[y, z] \\
\lambda[x, z] & =[\lambda x, z] \text { for all } x, y, z \in X \text { and } \lambda \text { scaler } \\
{[x, x] } & >0 \text { for all } x \neq 0 \\
|[x, y]|^{2} \leqq[x, x][y, y] &
\end{aligned}
$$

We call $X$ a semi-inner-product space (in short, s.i.p.s.). If $X$ is a s.i.p.s., one shows easily that $[x, x]^{1 / 2}$ is a norm on $X$. On the other hand, let $X$ be a normed space and $X^{*}$ its dual. For each $x \in X$, there exists by the Hahn-Banach theorem, at least one (and we shall choose one) functional $W x \in X^{*}$ such that $\langle x, W x\rangle=\|x\|^{2}$. Given any such mapping $W$ from $X$ into $X^{*}$ (ank in general, there are infinitely many such mappings), it is at once verified that $[x, y]=\langle x, W y\rangle$ defines a semi-inner-product (s.i.p.).

Definition. Given a linear transformation $T$ on a s.i.p.s., we call the set $W(T)=\{[T x, x]:[x, x]=1\}$ the numerical range of $T$.

An important fact concerning the notion of numerical range is the following [4, Th. 14]:

Let $X$ be a complex Banach space, and $T$ an operator on $X$. 
Although there may be many different s.i.p. consistent with the original norm of $X$, in the sense that $[x, x]=\|x\|^{2}$, nonetheless, the convex hulls of numerical range of $T$ relative to all such s.i.p. are equal. It has real numerical range with respect to one s.i.p., then it has real numerical range with respect to any other s.i.p. inducing the same norm.

DeFinition. Let $T$ be an operator on a complex Banach space $X$, then $T$ is called hermitian if its numerical range is real, relative to any s.i.p. consistent with the norm.

1. A general setting. We shall call an algebra $A$ over the complex field $C \mathrm{a}^{*}$-algebra if there is a mapping * defined on $A$ satisfying:

(i) $a \in A$ implies $a^{*} \in A$.

(ii) $(a+b)^{*}=a^{*}+b^{*}$ and $(\lambda a)^{*}=\bar{\lambda} a^{*}$.

(iii) $\left(a^{*}\right)^{*}=a$ and $(a b)^{*}=b^{*} a^{*}$ for all $a, b \in A$ and $\lambda \in C$. An element $a$ such that $a^{*}=a$ is said to be self-adjoint (s.a.). Every element $a$ of a ${ }^{*}$-algebra can be written in a unique way: $a=u+i v$ where $u$ and $v$ are s.a. A *algebra-isomorphism $\rho$ is an algebra isomorphism on a *algebra $A$ with the condition that $(\rho(a))^{*}=\rho\left(a^{*}\right)$ for all a in $A$.

Let $X$ be a complex s.i.p.s. and $A$ be a *algebra with a topology. Assume that $X$ is a two-sided module over $A$. Suppose that there is a net $\left\{e_{\alpha}\right\}$ in $A$ such that $\lim _{\alpha} f e_{\alpha}=f$ for all $f$ in $X$. For a *-subalgebra $A_{0}$ of $A$ such that $A_{0}$ is a subset of $X$, and $\left\{e_{\alpha}\right\}$ is contained in $A_{0}$, the following holds:

THEOREM 1. Suppose that for any s.a. $h$ in $A, H_{h} f=h f$ for all $f$ in $X$ defines a bounded hermitian operator on $X$; and that conversely every bounded hermitian operator is of this form. Then any onto isometry $U$ of $X$ when restricted to $A_{0}$ is given by

$$
U f=\lim _{\alpha} \rho(f) U \boldsymbol{e}_{\alpha}
$$

where $\rho$ is $a^{*}$-algebra-isomorphism on A.

Proof. Let $h$ in $A$ be s.a., then $H_{h}$ is a bounded hermitian operator on $X$. On the other hand, let a s.i.p. [,] on $X$ be given, then $[f, g]^{\prime}=\left[U^{-1} f, U^{-1} g\right]$ defines another s.i.p. on $X$ inducing the same norm. It follows that

$$
\left[U H_{h} U^{-1} f, f\right]^{\prime}=\left[H_{h} U^{-1} f, U^{-1} f\right] \text { is real for all } f \text {. }
$$

Thus $U H_{h} U^{-1}$ is another hermitian operator on $X$, and by hypothesis there is a s.a. $\hat{h}$ in $A$ such that 


$$
U H_{h} U^{-1} f=H_{\hat{h}} f \text { for all } f \text { in } X \text {. }
$$

Clearly the mapping $h \rightarrow \hat{h}$ is linear. If $\hat{h}=0$, then for all $f \in X$, $U H_{h} U^{-1} f=0$; in particular $U H_{h} U^{-1} U e_{\alpha}=U\left(h e_{\alpha}\right)=0$. Since $U$ is one to one, $h e_{\alpha}=0$ and $\lim _{\alpha} h e_{\alpha}=h=0$. Hence this mapping is one to one. We shall set $\rho(h)=\hat{h}$. With s.a. $h$ and $h^{\prime}$ in $A$,

$$
H_{\rho\left(h h^{\prime}\right)}=U H_{h h^{\prime}} U^{-1}=U H_{h} U^{-1} U H_{h^{\prime}} U^{-1}=H_{\rho(h)} H_{\rho\left(h^{\prime}\right)} .
$$

Thus $\rho\left(h h^{\prime}\right)=\rho(h) \rho\left(h^{\prime}\right)$. Extending $\rho$ on $A$ trivially by letting

$$
\rho\left(h+i h^{\prime}\right)=\rho(h)+i \rho\left(h^{\prime}\right),
$$

it can easily be shown that $\rho$ is a *algebra-isomorphism on $A$. For all $f$ in $A_{0}, U\left(f e_{\alpha}\right)=U H_{f} U^{-1} U e_{\alpha}=\rho(f) U e_{\alpha}$, so that

$$
U f=\lim _{a} \rho(f) U e_{\alpha} .
$$

2. Function spaces. Let $X$ be a Banach function space with absolutely continuous norm [6] over a $\sigma$-finite measure space $(\Omega, \Sigma, \mu)$.

Lemma 1. Assume that $\omega$ is a measurable subset of $\Omega$ and let $P$ be the projection of $X$ onto the subspace $E$ of functions in $X$ vanishing outside $\omega$. Then for any hermitian operator $H$ on $X$, PHP is a hermitian operator on $E$.

Proof. Since $X$ has absolutely continuous norm $X^{*}=X^{\prime}$, the associated space of $X$. Let $W$ be a mapping as before. Then a consistent s.i.p. on $X$ is given by: with each $g \in X$,

$$
[f, g]=\langle f, W g\rangle=\int f W g \text { for all } f \in X .
$$

Without loss of generality we can take $W g$ to be $\chi W g$ if $g \in E$ where $\chi$ is the characteristic function of $\omega$. Then for all $g \in E$ such that $\|g\|=1$, we obtain

$$
[H g, g]=\int H g \chi W g=\int \chi H g \chi W g=[(P H P) g, g]
$$

which is real valued. Thus $P H P$ is hermitian on $E$.

Lemma 2. [5, Lemma 7]. If $h \in L_{\infty}$ is a real function, the operator $H_{h}$, defined by $H_{h} f=h f$ for all $f \in X$, is a bounded hermitian operator on $X$; and $\left\|H_{k}\right\|=\|h\|_{\infty}$.

We shall use the following fact several times later. 
Lemma 3. For $\alpha, \beta, \gamma$ complex numbers such that $e^{2 \theta} \alpha+e^{-i \theta} \beta+\gamma$ is real for all $0 \leqq \theta<2 \pi$, then $\alpha=\bar{\beta}$ and $\gamma$ is real.

Let $E$ be a two-dimensional Banach space. Denote the element $f$ of $E$ as a function defined on the set $\Omega=\{x, y\}$. We shall assume that the norm in $E$ has the following properties:

(1) $\|f\|=\||f|\|$.

(2) $|f| \leqq|g|$ implies that $\|f\| \leqq\|g\|$ with all $f, g \in E$.

The real functions in $E$ can be considered as points in the twodimensional Euclidean plane; let $\gamma$ be the convex curve of the boundary of its real unit ball. At each point $p \in \gamma$ there is a supporting hyperplane, and suppose that the normal vector at $p$ to the hyperplane is given by $(\alpha, \beta)$. We shall define sgn $g$ as the function

$$
\operatorname{sgn} g= \begin{cases}0 & \text { if } g=0 \\ \frac{|g|}{g} & \text { otherwise. }\end{cases}
$$

LemMA 4. For any nonzero $g \in E$

$$
[f, g]=\|g\| A(g)\{f(x) \operatorname{sgn} g(x) \alpha(g)+f(y) \operatorname{sgn} g(y) \beta(g)\}
$$

where

$$
A(g)=\left\{\frac{|g(x)|}{\|g\|} \alpha(g)+\frac{|g(y)|}{\|g\|} \beta(g)\right\}^{-1} \text { and } \quad(\alpha(g), \beta(g))
$$

is a normal vector at $(|g(x)| /\|g\|,|g(y)| /\|g\|)$ for all $f \in E$, defines a consistent s.i.p. on $E$.

Proof. Clearly it is linear in $f$ and $[g, g]=\|g\|^{2}$. First we assume that $f$ and $g$ are real valued. The fact that $\|g\|=\||g|\|$ implies that the curve $\gamma$ is symmetric with respect to both axes. The function $A(g)\{s \alpha(g)+t \beta(g)\}$ has absolute value no greater than one on the region between the two lines $L_{1}$ and $L_{2}$ where they are two chosen supporting hyperplanes at $(|g(x)| /\|g\|,|g(y)| /\|g\|)$ and $(-|g(x)| /|| g||,-|g(y)| / \| g||)$ with normal vectors $(\alpha(g), \beta(g))$ and $(-\alpha(g),-\beta(g))$ respectively. So that $A(g)\|g\|\{|s \alpha(g)|+|t \beta(g)|\} \leqq\|g\|$ for all $(s, t) \in \gamma$. For all nonzero $f \in E,(|f(x)| /\|f\|,|f(y)| /\|f\|) \in \gamma$, we obtain

$$
A(g)\|g\|\{|f(x) \operatorname{sgn} g(x) \alpha(g)|+|f(y) \operatorname{sgn} g(y) \beta(g)|\} \leqq\|f\|\|g\| .
$$

Now in the above inequality, only the absolute values are involved, it holds for all complex functions $f$ and $g$ as well. 
Let $X_{n}$ be a $n$-dimensional real Banach space $(n \geqq 2)$ and $S$ its unit ball. We shall fix a basis for $X_{n}$ and denote every element $x$ as a point in the $n$-dimensional Euclidean space $E_{n}$. Define a function $F$ on $E_{n}$ as $F\left(x_{1}, x_{2}, \cdots, x_{n}\right)=\left\|\left(x_{1}, x_{2}, \cdots, x_{n}\right)\right\|-1$. For each $i=$ $1,2, \cdots n$, let $e^{i}=(0, \cdots, 0,1,0, \cdots 0)$ (1 at the $i$-th position).

Lemma 5. Let $S^{\prime}$ be an open set of $E_{n}$ consisting of smooth points of $X_{n}$, then the function $F$ has continuous first partial derivatives at every point of $S^{\prime}$.

Proof. If $x$ is a point of $S^{\prime}$, then the norm function is Gateaux differentiable at $x[7]$. Therefore with $i=1,2, \cdots, n$

$$
\lim _{t \rightarrow c} \frac{\left\|x+t e^{i}\right\|-\|x\|}{t}=\frac{\partial F}{\partial x_{i}}(x) .
$$

Suppose $W$ is as before, then from [5, Lemma 1]

$$
\|x\| \frac{\partial F}{\partial x_{i}}(x)=\left\langle e^{i}, W x\right\rangle=\left[e^{i}, x\right] .
$$

Since the norm topology of $X_{n}$ and that of $E_{n}$ are equivalent, the weak star compactness of the unit ball of $X_{*}^{*}$ and the smoothness of $S^{\prime}$ implies that this mapping $W$ is weak star continuous on $S^{\prime}$. Thus $\partial F / \partial x_{i}(x)$ is continuous on $S^{\prime}$.

Lemma 6. Let $H$ be a hermitian operator on $E$ and

$$
H=\left[\begin{array}{ll}
a & b \\
c & d
\end{array}\right] .
$$

Then either $b=c=0$ or else $b / \bar{c}>0$ and $E$ is a Hilbert space; in either case $a$ and $d$ are real numbers.

Proof. We shall start by proving that the set $S^{\prime}=\{(s, t): s \neq$ $0 \neq t\}$ consists of smooth points if $b$ and $c$ are not both zero. For $0 \leqq \theta \leqq 2 \pi$, let $f=\left(e^{2 \theta} s, t\right)$ be such that $(s, t) \in S^{\prime}$ and $\|f\|=1$, then by Lemma $4[H f, f]=A(f)\left(a s \alpha+d t \beta+e^{-i \theta} b t \alpha+e^{i \theta} c s \beta\right)$ is real, where $(\alpha, \beta)$ is the normal vector of a supporting hyperplane to the real unit ball $S$ at $(s, t)$. We have by Lemma 3 that

$$
\text { bt } \alpha-\bar{c} s \beta=0 \text {. }
$$

We assume that $c \neq 0$. If $b=0$ then $\beta=0$ for all such $f$ and $\gamma$ is a rectangle. As $\beta=0$ cannot occur on all four sides of a rectangle, $b$ and $c$ are not zero. $(\alpha, \beta)$ is uniquely determined up to a scaler multiple. Therefore the hyperplane is unique and every point of $S^{\prime}$ 
is smooth. Now for $b$ and $c$ being nonzero, the function $F(s, t)=$ $\|(s, t)\|-1$ is differentiable at $(s, t) \in S^{\prime}$. The hyperplane is thus given by the tangent plane. So that for all $g \in E$ such that $g(x) \neq$ $0 \neq g(y)$, the linear functional in Lemma 4 can be replaced by

$$
[f, g]=A(g)\|g\|\left\{f(x) \operatorname{sgn} g(x) \frac{\partial F}{\partial s}+f(y) \operatorname{sgn} g(y) \frac{\partial F}{\partial t}\right\}
$$

and we obtain the equation

$$
\frac{b t}{\bar{c}} \frac{\partial F}{\partial s}-s \frac{\partial F}{\partial t}=0 .
$$

Now $(b / \bar{c}) t^{2}+s^{2}$ satisfies the partial differential equation. By the uniqueness of solution, the curve $\gamma$ is given by the equation $s^{2}+(b / \bar{c}) t^{2}=$ $K$. Since the unit ball is bounded, $b / \bar{c}$ and $K$ must be positive. Then an inner-product on $E$ can be defined by

$$
(f, g)=\frac{f(x) \overline{g(x)}}{K}+\frac{b f(y) \overline{g(y)}}{\bar{c} K} .
$$

Thus $E$ is a Hilbert space.

For nonzero $g \in E$ such that $g(y)=0$, by Lemma $4[f, g]=$ $\|g\|^{2} f(x) / g(x)$ for all $f$ in $E$. As $[H g, g]=a\|g\|^{2}$ is real, $a$ is real; similarly $d$ is real.

3. Discrete symmetric Banach function spaces. Let $X$ be a Banach function space with absolutely continuous norm and the measure is purely atomic; so that $X$ is a sequence space. Assume that $X$ is symmetric, i.e., if $f$ in $X$ and $\phi$ is an isomorphism of the atoms, then $\|f\|=\|f(\phi)\|$. Choose the set of all characteristic functions of atoms to be a fixed basis for $X$. Let $H$ be a hermitian operator on $X$ and be represented as an infinite matrix $\left(a_{i j}\right)$, then Lemmas 1 and 6 imply that $a_{i j}=\bar{a}_{j i}$.

Lemma 7. If there is a hermitian operator $H$ on $X$ such that its matrix representation is not diagonal, then there is a hermitian operator $H^{\prime}$ on $X$ with all nonzero off diagonal entries.

Proof. We write $H=\left(a_{i j}\right)$. Assume that without loss of generality that $a_{12} \neq 0$; then $\bar{a}_{21} \neq 0$. Suppose that $i_{1}$ is the smallest positive integer such that $a_{1 i_{1}}=0$. Define $U_{1}$ on $X$ as operator obtained from the identity $I$ by interchanging its 2 nd and $i_{1}$-th row. Then $U_{1}$ is isometric and $H_{1}=U_{1} H U_{1}$ is hermitian. Choose $\alpha_{1}>0$ such that || $\alpha_{1} H_{1} \| \leqq 1 / 2$ and the matrix entries of $a_{1 j}$ of $H+\alpha_{1} H_{1}$ are nonzero for all $2 \leqq j \leqq i_{1}$. Assume that this has been done for $i_{n}$ steps and 
let $i_{n+1}$ be the smallest integer greater than $i_{n}$ such that $a_{1 i_{n+1}}=0$. Again let $H_{n+1}=U_{n+1} H_{n+1} U_{n+1}$ where $U_{n+1}$ is the isometric operator obtained from $I$ by interchanging the 2 nd and $i_{n+1}$-th row. Take $\alpha_{n+1}>0$ with $\left\|\alpha_{n+1} H_{n+1}\right\| \leqq 1 / 2^{n+1}$ and the matrix entries $a_{i j}$ of $H+\sum_{1 \leqq k \leqq n+1} \alpha_{k} H_{k}$ are not zero for $j=2, \cdots, i_{n+1}$. Then the operator $G_{1}=H+\sum_{k \geqq 1} \alpha_{k} H_{k}$ is a bounded hermitian on $X$. Its entries $a_{1 j} \neq 0$ for all $j \geqq 2$. With $i=2,3, \cdots$ let $V_{i}$ be the operator by interchanging first and $i$-th row of $I$. Then $G_{i}=V_{i} G_{1} V_{i}$ is hermitian and its entries $a_{i j} \neq 0$ for $j=1,2, \cdots, i-1, i+1, \cdots$. Choose a sequence $\left\{\beta_{i}\right\}$ of positive numbers such that $\sum_{\beta_{i}}<\infty$ and for each $k=2,3, \cdots$ the first $k$ rows of $\sum_{1 \leqq j \leqq k} \beta_{j} G_{j}$ are not zero except may be at the $(j, j)$ position. Then $H^{\prime}=\sum \beta_{j} G_{j}$ is the required hermitian operator.

Let $X_{n}=\{f \in X: f(k)=0$ all $k>n\}$. Suppose that $S$ is the real unit ball in $X_{n}$ as represented in the $n$-dimensional Euclidean space $E_{n}$ and $\gamma$ its boundary. For $\alpha \in \gamma$ there exists at least one supporting hyperplane to $S$ at $\alpha$ with a normal vector $\left(\alpha_{1}, \alpha_{2}, \cdots, \alpha_{n}\right)$.

Lemma 8. For nonzero $g \in X_{n}$,

$$
[f, g]=A(g)\|g\|\left\{\sum_{1 \leqq j \leqq n} f(j) \operatorname{sgn} g(j) \alpha_{j}\right\} \quad \text { all } f \in X_{n},
$$

where $A(g)=\left\{\sum_{j=1}^{n}|g(j)| /\|g\| \alpha_{j}\right\}^{-1}$ and $\left(\alpha_{1}, \alpha_{2}, \cdots, \alpha_{n}\right)$ is the normal vector to a hyperplane at $\left(\frac{|g(1)|}{\|g\|}, \frac{|g(2)|}{\|g\|}, \cdots, \frac{|g(n)|}{\|g\|}\right)$, defines a consistent s.i.p. on $X_{n}$.

The proof is similar to that as in Lemma 4 .

Lemma 9. If there is a $H^{\prime}$ as in Lemma 7, then the set $S^{\prime}=$ $\left\{f \in X_{n}: f(j) \neq 0\right.$ all $\left.j\right\}$ consists of smooth points.

Proof. Let $\left(x_{1}, x_{2}, \cdots, x_{n}\right) \in S^{\prime} \quad$ and $k=1,2, \cdots, n-1, g_{k}=$ $\left(x_{1}, x_{2}, \cdots, e^{i \theta} x_{k}, \cdots, x_{n}\right)$ in $X_{n}$ is of unit norm where $0 \leqq \theta<2 \pi$. The restriction of $H^{\prime}$ to $X_{n}, H_{n}=\left(a_{i j}\right)_{i, j=1,2, \ldots n}$ is hermitian by Lemma 1 and

$$
\begin{aligned}
{\left[H_{n} g_{k}, g_{k}\right]=} & A\left(g_{k}\right)\left\{\left(a_{11} x_{1}+\cdots+e^{i \theta} a_{1 k} x_{k}+\cdots+a_{1 n} x_{n}\right) \alpha_{1}+\cdots\right. \\
& +e^{-i \theta}\left(a_{k 1} x_{1}+\cdots+e^{i \theta} a_{k k} x_{k}+\cdots+a_{k n} x_{n}\right) \alpha_{k}+\cdots \\
& \left.+\left(a_{n 1} x_{1}+\cdots+e^{i \theta} a_{n k} x_{k}+\cdots+a_{n n} x_{n}\right) \alpha_{n}\right\} \\
= & A\left(g_{k}\right)\left\{e ^ { i \theta } \left(a_{1 k} x_{k} \alpha_{1}+\cdots+a_{k-1 k} x_{k} \alpha_{k}+a_{k+1 k} x_{k} \alpha_{k+1}+\cdots\right.\right. \\
& \left.+a_{n k} x_{k} \alpha_{n}\right)+e^{-i \theta}\left(a_{k 1} x_{1}+\cdots+a_{k k-1} x_{k-1}\right. \\
& \left.\left.+a_{k k+1} x_{k+1}+\cdots+a_{k n} x_{n}\right) \alpha_{k}+\cdots\right\}
\end{aligned}
$$


is real valued. By Lemma 3 we obtain the system of equations:

$$
\left(\sum_{\substack{j=1 \\ \neq k}}^{n} a_{k j} x_{j}\right) \alpha_{k}-\sum_{\substack{j=1 \\ \neq k}}^{n} \bar{a}_{j k} x_{k} \alpha_{j}=0
$$

for $k=1,2, \cdots, n-1$.

For every real number $\beta$, let $U$ be a diagonal matrix whose first diagonal element is $e^{-i \beta}$ and the rest is one. In place of $H^{\prime}$ we substitute $U H^{\prime} U^{-1}$. Then the resulting matrix elements are changed only for the first row and first column; and the subsequent form of equations (1) are:

$$
\begin{gathered}
\left(\sum_{j=2}^{n} a_{1 j} e^{-i \beta} x_{j}\right) \alpha_{1}-\sum_{j=2}^{n} \bar{a}_{j 1} e^{-i \beta} x_{1} \alpha_{j}=0 \\
\left(a_{k 1} e^{i \beta} x_{1}+\sum_{\substack{j=2 \\
\neq \neq}}^{n} a_{k j} x_{j}\right) \alpha_{k}-\left(\bar{a}_{1 k} e^{i \beta} x_{k} \alpha_{1}+\sum_{\substack{j=2 \\
\neq k}}^{n} \bar{a}_{j k} x_{k} \alpha_{j}\right)=0
\end{gathered}
$$

for $k=2,3, \cdots, n-1$. With any fixed $\left(x_{1}, x_{2}, \cdots, x_{n}\right)$ where $x_{j} \neq 0, j=$ $1,2, \cdots, n$, we shall show that this system is linearly independent for some $\beta$; equivalently we show that the following matrix is rank $n-1$ :

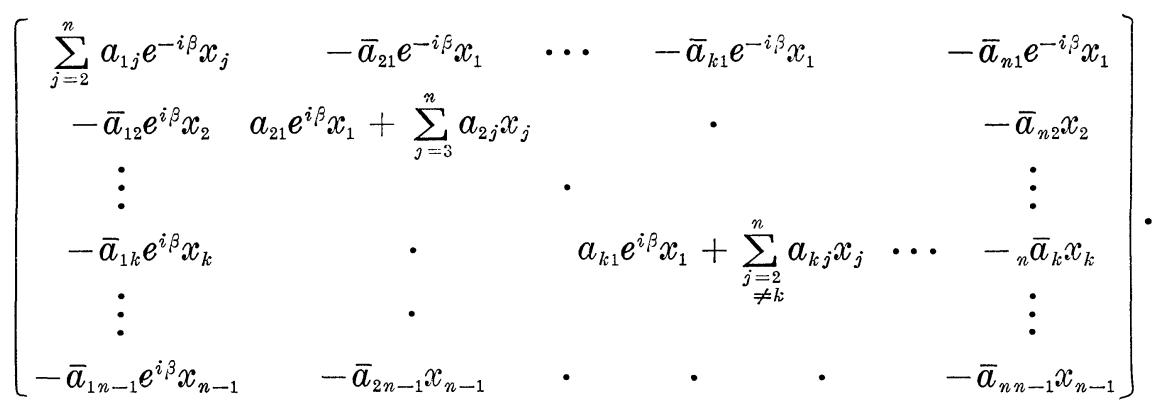

If we take the first $n-1$ columns, we obtain a square matrix and its determinant is a polynomial $P\left(e^{i \beta}\right)$ of degree $n-2$. The coefficient of the $e^{i(n-2), s}$ term is obtained by finding the determinant of the following matrix:

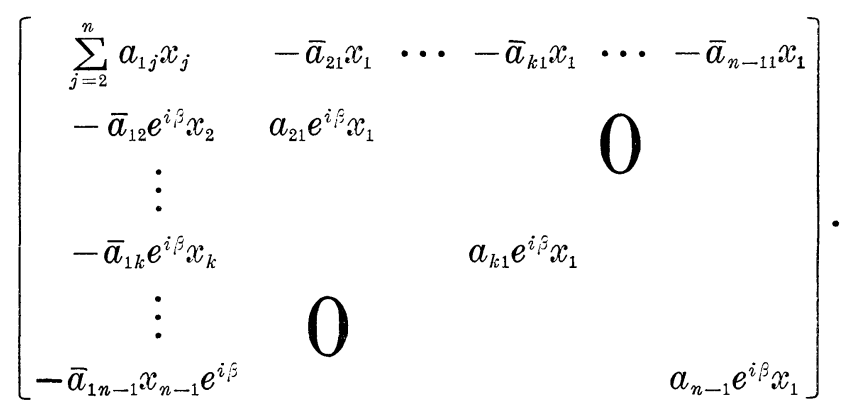

For $k=2,3, \cdots, n-1$, we add $\bar{a}_{k 1} e^{-i \beta} / a_{k 1}$ multiple of $k$-th row to the first row. We obtain by the condition that $a_{1 k}=\bar{a}_{k 1}$ a matrix of non- 
zero diagonal elements and whose entries above diagonal are zero. Thus the polynomial $P$ is not identically zero and the original matrix has rank $n-1$ for some $\beta$. Thus we may assume that the system (1) is linearly independent. This implies that the normal vector $\left(\alpha_{1}, \alpha_{2}, \cdots, \alpha_{n}\right)$ is uniquely determined up to a multiple of constant. The proof is complete.

THEOREM 2. Suppose that $H$ is a hermitian operator on $X$, then either there is real valued function $h \in l_{\infty}$ such that

$$
H f=h f \quad \text { for all } f \in X
$$

and $\|H\|=\|h\|_{\infty}$ or else $X$ is a Hilbert space. Conversely for every real valued function $h \in l_{\infty}$ the above formula defines a hermitian operator on $X$.

Proof. The converse is the content of Lemma 2. Assume that there is a hermitian operator $H$ on $X$ which is not diagonal, then Lemmas 7 and 9 imply that the function $F$ defined on $E_{n}$, given by $F\left(x_{1}, x_{2}, \cdots, x_{n}\right)=\left\|\left(x_{1}, x_{2}, \cdots, x_{n}\right)\right\|-1$, is differentiable at points of $S^{\prime}$. So that the supporting hyperplane at $g \in S^{\prime}$ is given by tangent place and the system (1) can be replaced by

$$
\sum_{\substack{j=1 \\ \neq k}}^{n} a_{k j} x_{j} \frac{\partial F}{\partial x_{k}}-\sum_{\substack{j=1 \\ \neq \neq}}^{n} \bar{a}_{j k} x_{k} \frac{\partial F}{\partial x_{j}}=0
$$

$k=1,2, \cdots, n-1$. Observe that the function $\sum_{i=1}^{n} x_{i}^{2}$ satisfies this system. Let $x^{0}=\left(x_{1}^{0}, x_{2}^{0}, \cdots, x_{n}^{0}\right)$ be a point on the unit ball and $\sum_{i=1}^{n}\left(x_{i}^{0}\right)^{2}=K$ for some $K>0$. For all other $x \in S^{\prime}$ which is on this sphere we have

$$
F(x)=F\left(x^{0}\right)+\int_{\Gamma} \operatorname{grad} F=\int_{\Gamma} \sum_{i=1}^{n} \frac{\partial F}{\partial x_{i}} \frac{d x_{i}}{d s} d s
$$

where $T=\left(d x_{1} / d s, d x_{2} / d s, \cdots, d x_{n} / d s\right)$ is the unit tangent vector. If $F(x) \neq 0$, since $\operatorname{grad} F . T$ is continous, then there is a $s_{0}$ such that $x\left(s_{0}\right) \in \Gamma$ and grad $F\left(s_{0}\right) . T\left(s_{0}\right) \neq 0$. But $T\left(s_{0}\right)$ at $x\left(s_{0}\right)$ is on the tangent plane to the sphere at $x\left(s_{0}\right)$ and grad $F\left(s_{0}\right)$ is normal to this plane, this is a contradiction. Therefore $F(x)=0$ and all $x \in S^{\prime}$ such that $\sum_{i=1}^{n} x_{i}^{2}=K$ are on the real unit ball. As the surface $\gamma$ is continuous, this equation gives the set of points on $\gamma$.

This will suffice to imply that $X_{n}$ is a Hilbert space, since an inner-product on it can be found to give the original norm. The absolute continuity of the norm thus implies that $X$ is a Hilbert space. 
If $X$ is not a Hilbert space, then every $H$ on $X$ is real diagonal and the rest is clear.

THEOREM 3. Suppose $U$ is an isometry from $X$ onto itself and assume that $X$ is not a Hilbert space. Then there is a fixed unimodular function $u$ and an isomorphism $T$ of atoms such that

$$
U f(.)=u(.) f(T .) \quad \text { for all } f \in X .
$$

Conversely such a transformation always defines as isometry on $X$.

Proof. The line of argument follows that of Theorem 5 below. $u$ is unimodular because of the symmetry condition on $X$.

4. Reflexive Orlicz spaces. Let $L_{M \Phi}$ be a reflexive Orlicz space defined by the convex function $\Phi$. We assume that $\Phi$ is everywhere finite. Suppose that the measure is finite.

Lemma 10. [5, Lemma 6]. Let $H$ be a bounded hermitian operator on $L_{M \Phi}$. If $\Omega^{\prime}, \Omega^{\prime \prime}$ are a.e. disjoint, i.e., $\mu\left(\Omega^{\prime} \cap \Omega^{\prime \prime}\right)=0$, let $\chi^{\prime}$ and $\chi^{\prime \prime}$ be their characteristic functions; then $\int_{\Omega^{\prime}} H \chi^{\prime \prime}=0$ if and only if $\int_{\Omega^{\prime \prime}} H \chi^{\prime}=0$.

Lemma 11. [5, Th. 9]. Suppose $H$ is as above, and $\mu$ is purely nonatomic, then either there exists a real valued function $h \in l_{\infty}$ such that $H f=h f$ for all $f \in L_{M}$ and $\|H\|=\|h\|_{\infty}$ or else $L_{M \Phi}=L_{2}$.

Let $(\Omega, \Sigma, \mu)$ be a general measure space and decompose $L_{M \Phi}=$ $L_{M \Phi}^{\prime}+l_{M \Phi}$ where $L_{M \Phi}^{\prime}$ are functions on nonatomic part and $l_{M \Phi}$ are functions on purely atomic part.

Lemma 12. Suppose $H$ is as above, then either $L_{M \Phi}$ is $L_{2}$ or else $L_{M \Phi}^{\prime}$ and $l_{M \Phi}$ are both invariant under $H$.

Proof. Assume that $L_{M \Phi}$ is not a $L_{2}$ space. Let $\Omega^{\prime}$ be a nonzero atom and $\chi^{\prime}$ its characteristic function. Suppose that $H \chi^{\prime}$ is not zero on a nonatomic set $\Omega^{\prime \prime}$, and $\int_{\Omega^{\prime \prime}} H \chi^{\prime} \neq 0$. Take $\chi^{\prime \prime}$ to be the characteristic function of $\Omega^{\prime \prime}$. Then for $\alpha \geqq 0$ we obtain the equality as in the proof of Lemma 11 [see 5]:

$$
\Psi\left(\frac{\alpha}{\left\|\alpha \chi^{\prime \prime}+\chi^{\prime}\right\|}\right)=\alpha \Psi\left(\frac{1}{\left\|\alpha \chi^{\prime \prime}+\chi^{\prime}\right\|}\right)
$$

where $\Psi=1 / 2\left(\Phi^{+}+\Phi^{-}\right)$and $\Phi^{+}, \Phi^{-}$are the right and left hand derivatives of $\Phi$ respectively. Since $\Omega^{\prime \prime}$ is nonatomic, we may replace 
$\Omega^{\prime \prime}$ by subset of $\Omega^{\prime \prime}$ with arbitrarily small measure, so that

$$
\Psi\left(\frac{\alpha}{\left\|\chi^{\prime}\right\|}\right)=\alpha \Psi\left(\frac{1}{\left\|\chi^{\prime}\right\|}\right) \quad \alpha \geqq 0 .
$$

Then $\Phi(t)=c t^{2}$ and $L_{M \Phi}$ is actually a $L_{2}$ space. This contradict our hypothesis, $H \chi^{\prime} \in l_{M \Phi}$.

Conversely, if $\Omega^{\prime \prime}$ is nonatomic and $\chi^{\prime \prime}$ its characteristic function, then by Lemma $10, \int_{\Omega^{\prime}} H \chi^{\prime \prime}=0$ if and only if $\int_{\Omega^{\prime \prime}} H \chi^{\prime}=0$ where $\Omega^{\prime}$ is any atom. The previous result shows that $H \chi^{\prime} \in l_{M \Phi}$ for every atom $\Omega^{\prime}$. Hence $\int_{\Omega^{\prime}} H \chi^{\prime \prime}=0$. Therefore $H \chi^{\prime \prime} \in L_{M \Phi}^{\prime}$. Since the step functions are dense in their respective subspaces, both $L_{M \Phi}^{\prime}$ and $l_{M \Phi}$ are invariant under $H$.

THEOREM 4. Suppose $H$ is a bounded hermitian operator on $L_{M \Phi}$ which is not a $L_{2}$ space, then one of the following three cases holds:

(1) $l_{M \Phi}$ is a Hilbert space.

(2) $l_{M \Phi}$ contains a two-dimensional Hilbert space but is not a Hilbert space.

(3) There is a fixed real valued function $h \in l_{\infty}$ such that $H f=$ $h f$ for all $f \in L_{M \oplus}$ and $\|H\|=\|h\|_{\infty}$.

Proof. By Lemma 12 and Lemma 11 it is enough to consider the restriction $H^{\prime}$ of $H$ on $l_{M \Phi}$. If $l_{M \Phi}$ does not have a two-dimensional Hilbert subspace, the $H^{\prime}$ is real diagonal by Lemma 6 and case (3) follows.

REMARK. Let $\mu$ be a $\sigma$-finite measure and $Q=\bigcup_{n=1}^{\infty} \Omega_{n}$ where $\left\{\Omega_{n}\right\}$ is a fixed increasing sequence of measurable sets with finite mass. Suppose that for each $n, P_{n}$ is the projection onto the subspace $X_{n}$ of functions restricted to $\Omega_{n}$. $H_{n}=P_{n} H P_{n}$ is hermitian. As $L_{M \Phi}$ has absolutely continuous norm, we have for $g \in L_{M \Phi}\left\|H g-H P_{n} g\right\| \rightarrow 0$ as $n \rightarrow \infty$, and $\left\|H g-H_{n} g\right\| \leqq\left\|H g-H P_{n} g\right\|+\left\|H P_{n} g-H_{n} g\right\|$, so that $H g=\lim _{n} H_{n} g$. Thus we show that Theorem 4 holds for $\sigma$-finite measure as well.

Let $L_{M \Phi}^{b}$ be the set of all $f \in L_{M \Phi} \cap L_{\infty}$. $L_{\infty}$ forms a *-algebra under the ordinary conjugation with the set of elements $\left\{\chi_{n}: \chi_{n}\right.$ characteristic functions of $\left.\Omega_{n}\right\}$ satisfying $\lim _{n} f \chi_{n}=f$ for all $f \in L_{M \phi} . \quad L_{M \Phi}^{b}$ contains this sequence. Suppose that $l_{M \Phi}$ is not a Hilbert space and contains no Hilbert subspace. Then the following is true.

Lemma 13. Suppose that $U$ is an isometry of $L_{M \Phi}$. Then there is $a^{*}$-isomorphism $\rho$ on $L_{\infty}$ such that $U g=u \rho(g)$ for all $g \in L_{M \Phi}^{b}$, where $u \neq 0$ a.e. 
Proof. By Theorem 1 and Theorem 4 we have for all $g \in L_{M \Phi}^{b}$, $\lim _{n} \rho(g) U \chi_{n}=U g$. It is enough to show that $U \chi_{n}$ converges a.e. to a nonzero function $u$. Since $\rho$ is an isomorphism, it sends characteristic functions onțo themselves. Define $T \omega=\omega^{\prime}$, where $\rho\left(\chi_{\omega}\right)=\chi_{\omega^{\prime}}$. For every $n \geqq 1, U H_{\chi_{n}} U^{-1}=H_{\rho\left(\chi_{n}\right)}=H_{\chi_{T \Omega_{n}}}$, so that $U\left(\chi_{n}\right)=\chi_{T \Omega_{n}} U \chi_{n}$. That is $U \chi_{n}=0$ on $\Omega-T \Omega_{n}$. Similarly $U\left(\chi_{\Omega_{n}-\Omega_{m}}\right)$ vanishes on $\Omega-T\left(\Omega_{n}-\Omega_{m}\right)=\Omega-\left(T \Omega_{n}-T \Omega_{m}\right)$ for $1 \leqq m \leqq n$; therefore $U \chi_{n}=U \chi_{m}$ on $T \Omega_{n}$ and $\lim _{n} U \chi_{n}=u$ exists a.e.

Assume that $\omega$ is a measurable subset of $T \Omega$ such that $0<\mu(\omega)<$ $\infty$ and $u=0$ on $\omega$. For every $h \in L_{M \perp}^{b}, U h=u \rho(h)=0$ on $\omega . L_{M \Phi}^{b}$ is dense in $L_{M \Phi}$, so that with every $f \in L_{M \Phi}$, there is a sequence $\left\{f_{n}\right\}$ in $L_{M \Phi}^{b}$ such that $f_{n} \rightarrow f$ as $n \rightarrow \infty$. Since the norm is absolutely continuous, there is a subsequence $\left\{f_{n_{k}}\right\}$ such that $U f_{n_{k}} \rightarrow U f$ a.e. Thus $U f=0$ on $\omega$. But $U$ is onto and $\chi_{\omega}$ is in the range of $U$. Hence $u$ is nonzero a.e.

DeFinition. A regular set isomorphism of a measure space $(\Omega, \Sigma, \mu)$ will mean a mapping $S$ of $\Sigma$ into $\Sigma$ defined modulo set of measure zero, satisfying: (i) $S(\Omega-\omega)=S \Omega-S \omega$. (ii) $S\left(\bigcup_{n=1}^{\infty} \omega_{n}\right)=\bigcup_{n=1}^{\infty} S \omega_{n}$ for disjoint sets $\left\{\omega_{n}\right\}$. (iii) $\mu(\omega)=0$ if and only if $\mu(S \omega)=0$.

Lemma 14. T, defined as in the proof above, is a regular set isomorphism of the underlying measure space; and it induces a linear transformation on $L_{M \varphi}\left(f(.) \rightarrow f\left(T^{-1}.\right)\right)$.

Proof. It is routine to show that $T$ is regular. Let $f \in L_{M \Phi}$ be $a \leqq f<b$ on a measurable set $\omega$ and zero elsewhere. Assume that $\left\{f_{n}\right\}$ is a sequence of step functions whose values lying between $a$ and $b$ on $\omega$ and zero elsewhere, such that $f_{n} \rightarrow f$ as $n \rightarrow \infty$. Then $U f_{n}=$ $u \rho\left(f_{n}\right)$ converges to $U f=u \rho(f)$ as $n \rightarrow \infty$. There is a subsequence $u \rho\left(f_{n_{k}}\right)$ converging to $u \rho(f)$ a.e. Since $u \neq 0, \rho\left(f_{n_{k}}\right) \rightarrow \rho(f)$ a.e. We denote the step function $\rho\left(f_{n_{k}}\right)$ as $f_{n_{k}}\left(T^{-1}.\right)$. Then $a \leqq f_{n_{k}}\left(T^{-1}.\right)<b$ on $T \omega ; \rho(f)$, the a.e. limit of $f_{n_{k}}\left(T^{-1}.\right)$, has the same property. We shall let this function be $g$. For any nonnegative function $f$ of $L_{M \Phi}$, let $\omega_{n}=\{x: n \leqq f(x)<n+1\}$ and $f_{n}$ be the restriction of $f$ to $\omega_{n}$. Then $g_{n}$ is $n \leqq g_{n}<n+1$ on $T \omega_{n}$ and zero elsewhere. Since $T$ is regular, we can compose these functions to be a function $g$; and denote it by $f\left(T^{-1}\right.$.). Extend this definition to negative and then complex functions. The mapping so defined is clearly linear.

Combining the results, we obtain the following isometry theorem:

THeOREM 5. Let $U$ be an isometry from a reflexive Orlicz space $L_{M \Phi}=L_{M \Phi}^{\prime}+l_{M \Phi}$ onto itself. Suppose that $L_{M \Phi} \neq L_{2}$, then $U$ can be 
decomposed into $U_{1}+U_{2}$ where $U_{1}$ and $U_{2}$ are isometric on $L_{M \Phi}^{\prime}$ and $l_{M \Phi}$ respectively. Moreover one of the following three cases holds.

(1) $l_{M \Phi}$ is a Hilbert space.

(2) $l_{M \Phi}$ is not a Hilbert space but contains a two-dimensional Hilbert subspace.

(3) There is a regular set isomorphism $T$ of the underlying measre space and a fixed a.e. nonzero function $u$ such that

$$
U f(.)=u(.) f\left(T^{-1} .\right) \quad \text { for all } f \in L_{M \oplus} .
$$

Proof. We first show that $U$ decomposes. For all real function $h \in L_{\infty}, U^{-1} H_{h} U L_{M \oplus}^{\prime} \subseteq L_{M \oplus}^{\prime}$ by Lemma 12 . Hence $H_{h} U L_{M \oplus}^{\prime} \subseteq U L_{M \Phi}^{\prime}$. If $U L_{M \Phi}^{\prime} \nsubseteq L_{H \Phi}^{\prime}$, then there is a characteristic function $\chi$ of some atom $\{a\}$ such that $U g=\chi$ with some $g$ in $L_{M \varphi}^{\prime}$. Without loss of generality we may assume that $g$ is a characteristic function of a nonatomic set $\omega$. For two disjoint sets $\omega^{\prime}, \omega^{\prime \prime}$ and $\chi^{\prime}, \chi^{\prime \prime}$ their characteristic functions, $\left\|U\left(\chi^{\prime}+\alpha \chi^{\prime \prime}\right)\right\|=\left\|U \chi^{\prime}+\alpha U \chi^{\prime \prime}\right\|=\left\|\chi^{\prime}+\chi^{\prime \prime}\right\|$ where $|\alpha|=1$ and $\omega=\omega^{\prime} \cup \omega^{\prime \prime}$. Thus $U \chi^{\prime}$ and $U \chi^{\prime \prime}$ cannot be both nonzero at $\{a\}$. Since $\omega$ is nonatomic, we may replace it by subset of arbitrarily small measure; $U g=0$. This contradicts the fact that $\chi \neq 0$. Hence $U L_{M \oplus}^{\prime} \subseteq$ $L_{M \Phi}^{\prime}$; simiarly $U^{-1} L_{M \Phi}^{\prime} \subseteq L_{M \Phi}^{\prime} . U\left(L_{M \Phi}^{\prime}\right)=L_{M \Phi}^{\prime}$. It follows that $U l_{M \Phi} \subseteq l_{M \Phi}$ with an application of Lemma 12.

Now if $l_{M \Phi}$ is not a Hilbert space and does not contain a twodimensional Hilbert subspace, then Lemma 2 and Theorem 4 imply that $H$ is a hermitian operator on $L_{M \Phi}$ if and only if it is of the form as stated in case (3) of Theorem 4. Hence case (3) holds for all $g$ in $L_{M \Phi}^{b}$ by Lemma 13 and Lemma 14. Since $L_{M \Phi}^{b}$ is dense, the proof is thus complete.

As a special case of the theorem, we record the following result as a corollary.

COROLlary. With the conditions as before and assume that the atoms in the measure space have equal mass, either

(1) There is a regular set isomorphism $T$ and a fixed a.e. nonzero function $u$ such that $U f()=.u() f.\left(T^{-1}\right.$.) all $f$ in $L_{M \Phi}$, or else

(2) $U_{1}$ is of the form as stated in (1) ( $T$ and $u$ in this case are defined only on the nonatomic part) and $U_{2}$ is unitary on $l_{M \oplus}$ which is a Hilbert space.

Remark. $U_{1}$ is always characterized in (3) of the Theorem 5 if $L_{M \Phi}$ is not a $L_{2}$ space.

5. An example. The following example shows that the Theorem 
3 does not hold if the symmetry condition is not present. It also shows that isometries other than the type in Theorem 5 occur if the atoms in the underlying measure space have unequal mass.

Let $(\Omega, \Sigma, \mu)$ be a measure space with contains two atomic sets $m_{1}$ and $m_{2}$ each with measure 16 and at least one other measurable set $m_{3}$ of mass 1 . With $\Phi(x)=\int_{0}^{x} \psi(t) d t$ where

$$
\psi(t)= \begin{cases}2 t & 0 \leqq t<1 / 2 \\ t^{2}+3 / 4 & t>1 / 2\end{cases}
$$

the obtained $L_{M \Phi}$ is not a Hilbert space. Specifically the two dimensional subspace on $\left\{m_{2}, m_{3}\right\}$ is not a Hilbert space, because the convex curve $\{(y, z): 16 \Phi(|y|)+\Phi(|z|)=1\}$ is not an ellipse. Now write $L_{M \Phi}=$ $l_{1}+l_{2}$ where $l_{2}$ is the two dimensional space of functions vanishing on $\Omega-\left\{m_{1}, m_{2}\right\}$ and $l_{1}$ of those being zero on $\left\{m_{1}, m_{2}\right\}$. Define $U=U_{1}+U_{2}$ where $U_{2}$ on $l_{2}$ in matrix form is

$$
U_{2}=\left(\begin{array}{ll}
\frac{1}{\sqrt{2}} & \frac{1}{\sqrt{2}} \\
-\frac{1}{\sqrt{2}} & \frac{1}{\sqrt{2}}
\end{array}\right)
$$

and $U_{1}$ is identity on $l_{1}$. Then for any $L_{M \oplus}$ such that $\|f\|=1$, we have $0 \leqq\left|f\left(m_{1}\right)\right|,\left|f\left(m_{2}\right)\right| \leqq 1 / 4$, so that

$$
\begin{aligned}
& \int \Phi(|U f|)=16\left\{\Phi\left(\left|U f\left(m_{1}\right)\right|\right)+\Phi\left(\left|U f\left(m_{2}\right)\right|\right)\right\}+\int_{\Omega-\left\{m_{1} \cdot m_{2}\right\}} \Phi(|U f|) \\
& =16\left\{\left|f\left(m_{1}\right)\right|^{2}+\left|f\left(m_{2}\right)\right|^{2}\right\}+\int_{\Omega-\left\{m_{1}, m_{2}\right\}} \Phi(|U f|)=\int \Phi(|f|) .
\end{aligned}
$$

Therefore $\|U f\|=\|f\|=1 . \quad U$ is isometric.

\section{BIBLIOGRAPHY}

1. S. Banach, Theorie des operations lineaires, Warszwa, 1932.

2. M. A. Kranoselski and Ya. Rutichki, Convex functions and Orlicz spaces, English translation, Grouingen, 1961.

3. J. Lamperti, On the isometries of certain function spaces, Pacific J. Math. 8 (1958), 451-466.

4. G. Lumer, Semi-inner-product spaces, Trans. Amer. Math. Soc. 100 (1961), 29-43.

5. - On the isometries of reflexive Orlicz spaces, Ann. Inst. Fourier, Grenoble 13 (1963), 99-100.

6. W. A. J. Luxemburg, Banach functions spaces, Thesis, 1955.

7. S. Mazur, Uber schwache Konvergenz in den Raumen $\left(L_{p}\right)$. Studia Math. 4 (1933), 128-133.

Received October 9, 1967, and in revised form May 8, 1969.

UNIVERSITY OF WASHINGTON AND

Portland State University 


\section{PACIFIC JOURNAL OF MATHEMATICS}

\section{EDITORS}

H. ROYDEN

Stanford University

Stanford, California

Richard Pierce

University of Washington

Seattle, Washington 98105
J. DUGUNDJI

Department of Mathematics

University of Southern California

Los Angeles, California 90007

BASIL GORDON

University of California

Los Angeles, California 90024

\section{ASSOCIATE EDITORS}
E. F. BECKENBACH
B. H. NEUMANN
F. WOLF
K. YosHIDA

\section{SUPPORTING INSTITUTIONS}

UNIVERSITY OF BRITISH COLUMBIA

CALIFORNIA INSTITUTE OF TECHNOLOGY

UNIVERSITY OF CALIFORNIA

MONTANA STATE UNIVERSITY

UNIVERSITY OF NEVADA

NEW MEXICO STATE UNIVERSITY

OREGON STATE UNIVERSITY

UNIVERSITY OF OREGON

OSAKA UNIVERSITY

UNIVERSITY OF SOUTHERN CALIFORNIA
STANFORD UNIVERSITY

UNIVERSITY OF TOKYO

UNIVERSITY OF UTAH

WASHINGTON STATE UNIVERSITY

UNIVERSITY OF WASHINGTON

*

AMERICAN MATHEMATICAL SOCIETY CHEVRON RESEARCH CORPORATION TRW SYSTEMS

NAVAL WEAPONS CENTER 


\section{Pacific Journal of Mathematics}

\section{Vol. 31, No. $1 \quad$ November, 1969}

James Burton Ax, Injective endomorphisms of varieties and schemes........

Richard Hindman Bouldin, A generalization of the Weinstein-Aronszajn

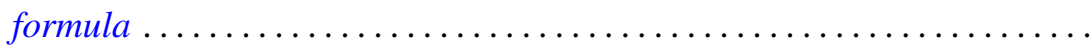

John Martin Chadam, The asymptotic behavior of the Klein-Gordon equation with external potential. II ...............................

Rina Hadass, On the zeros of the solutions of the differential equation

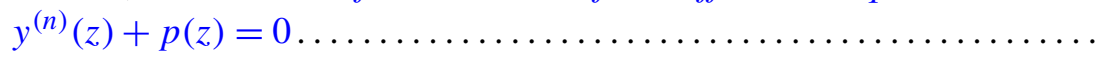

John Sollion Hsia, Integral equivalence of vectors over local modular lattices. II .............................................

Robert Hughes, Boundary behavior of random valued heat polynomial

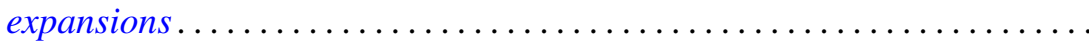

Surender Kumar Jain, Saad H. Mohamed and Surjeet Singh, Rings in which every right ideal is quasi-injective .........................

T. Kawata, On the inversion formula for the characteristic function .........

Erwin Kleinfeld, On right alternative rings without proper right ideals......

Robert Leroy Kruse and David Thomas Price, On the subring structure of

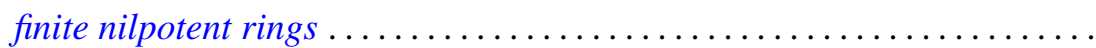

Marvin David Marcus and Stephen J. Pierce, Symmetric positive definite multilinear functionals with a given automorphism. .

William Schumacher Massey, Pontryagin squares in the Thom space of a bundle...

William Schumacher Massey, Proof of a conjecture of Whitney ...

John William Neuberger, Existence of a spectrum for nonlinear transformations

Stephen E. Newman, Measure algebras on idempotent semigroups ...

$\mathrm{K}$. Chandrasekhara Rao, Matrix transformations of some sequence spaces

Robert Bruce Schneider, Some theorems in Fourier analysis on symmetric

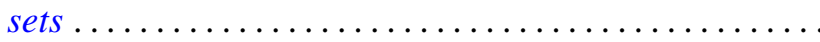

Ulrich F. K. Schoenwaelder, Centralizers of abelian, normal subgroups of hypercyclic groups...

Jerrold Norman Siegel, $G$-spaces, $H$-spaces and $W$-spaces

Robert Irving Soare, Cohesive sets and recursively enumerable Dedekind cuts...

Kwok-Wai Tam, Isometries of certain function spaces .... . .

Awadhesh Kumar Tiwary, Injective hulls of semi-simple modules over regular rings ....

Eldon Jon Vought, Concerning continua not separated by any nonaposyndetic subcontinuum .................... 